\title{
AN EXAMPLE CONCERNING ALGEBRAS OF MEASURABLE FUNCTIONS ${ }^{1}$
}

\section{ANTHONY W. HAGER}

If $A$ is a point-separating $\sigma$-algebra of subsets of the set $S$, a function $f: S \rightarrow R(R=$ the reals $)$ is called $\mathcal{A}$-measurable if $f \leftarrow(I) \in \mathcal{A}$ for each interval $I$. The family of such functions can be equipped with pointwise addition, multiplication, and multiplication by real numbers, and becomes what we shall call an algebra of measurable functions on the set $S$. Such an algebra $A$ has many properties, among them: $A$ is closed under uniform convergence on $S$, and is regular in the sense of von Neumann (i.e., given $f$ there is $g$ with $f^{2} g=f$ ). In fact, these two properties characterize such algebras. That is: Let A be a point-separating subalgebra of $R^{\mathrm{S}}$, with $1 \in A$. If $\mathrm{A}$ is regular and closed under uniform convergence, then $A$ is an algebra of measurable functions on $S$. (This observation seems essentially due to B. Brainerd. This is discussed, and other similar results surveyed, in [4].) In the present context, the proof of this is not hard; we sketch it: since $A$ is closed under uniform convergence, it is a lattice and the family $A \equiv\{\mathrm{coz}(f): f \in A\}$ is closed under countable union (where $\operatorname{coz}(f)=\{s \in S: f(s) \neq 0\}$ ); since $A$ is regular, $A$ is closed under complementation, and is an $\sigma$-algebra; easy approximation techniques now show that $A$ coincides with the algebra of $A$-measurable functions on $S$.

This note concerns the question of whether or not a certain weakening of the hypotheses in the above theorem entails the conclusion. We formulate the question in the following paragraphs. To avoid repetition, from now on each $A$ is to be a point-separating subalgebra of some $R^{\text {s }}$, with $1 \in A$.

Evidently, if $A$ is regular, then $A$ is closed under inversion (of functions without zeros). And, if $A$ is regular and closed under uniform convergence, then $A$ induces a (completely regular Hausdorff) " $P$ space" topology on $S$, i.e., one in which whenever a $G_{\delta}$-set contains a point, it is a neighborhood of the point. This can be proved directly, but it is seen more easily if we assume (via the above theorem) that $A$ is the algebra of $A$-measurable functions; then $\mathcal{A}$ is an open basis which is closed under countable intersection.

Received by the editors July 25, 1969.

AMS 1970 subject classifications. Primary 06A70, 28A20, 54C30; Secondary 06A40, 46A30, 46E $25,46 \mathrm{E} 30,54 \mathrm{C} 40$.

${ }^{\mathrm{l}}$ This research was partially supported by a grant from Wesleyan University, and by the National Science Foundation of the United States under grant GP-18825. 
A preliminary version of our question reads: is $A$ an algebra of measurable functions on $S$ if $A$ is closed under inversion, closed under uniform convergence, and induces a $\boldsymbol{P}$-space topology on $S$ ? The answer here is "no," and this is fairly easy to see. Let $S$ be an uncountable set, and $A$ the algebra of functions which are continuously extendible over $\alpha S$, the one-point compactification of $S$. Here $A$ induces the discrete topology on $S$, and the other properties are trivial. But if $\left\{p_{1}, p_{2}, \cdots\right\}$ is a countably infinite subset of $S$, and $f\left(p_{n}\right)=1 / n$ while $f(p)=0$ if $p \neq p_{n}$ for each $n$, then $f \in A$, but there is no $g \in A$ with $f^{2} g=f$. Thus $A$ is not regular, and cannot be an algebra of measurable functions. This example makes it clear that our question is not the right one: we ought to be considering $A$ as a subalgebra of $R^{\alpha S}$; but on $\alpha S, A$ induces the usual compact topology and this is not a $P$-space topology.

We sharpen our view of the situation. Let $A$ be a point-separating subalgebra of $R^{\mathrm{s}}$, with $1 \in A$, and suppose for simplicity that $A$ is closed under uniform convergence. Observe that, if $p \in S$, then

$$
M_{p} \equiv\{f \in A: f(p)=0\}
$$

is a maximal ideal of $A$ and $A / M_{p}$ is isomorphic to $R$. Let $\mathcal{R}(A)$ be the set of all maximal ideals $M$ with $A / M$ isomorphic to $R$, equipped with the hull-kernel topology. For $f \in A$, define the continuous function $f^{\prime}: \mathcal{R}(A) \rightarrow R$ by $f^{\prime}(M)=f+M$. Then $A^{\prime} \equiv\left\{f^{\prime}: f \in A\right\}$ is isomorphic to $A$, is a point-separating subalgebra of $R^{\mathcal{R}(A)}$, and the topology on $\mathcal{R}(A)$ which $A^{\prime}$ induces is the hull-kernel topology. (See, e.g., [1, Theorem 4.3], or some of the references in [4].) It is now a simple matter to prove that $S$, with the topology induced by $A$, embeds homeomorphically and densely in $\mathscr{R}(\boldsymbol{A})$.

Thus, the following would appear to be the proper formulation of our question. Observe that the condition on ideals asserts the maximality of $S$ with respect to the other hypotheses.

Question. ${ }^{2}$ Let $A$ be a point-separating subalgebra of $R^{\mathrm{S}}$, with $1 \in A$, which is closed under inversion of functions without zeros and uniform convergence on $S$, which induces a $P$-space (or even discrete) topology on $S$ and for which each maximal ideal $M$ with $A / M=R$ is of the form $\{f \in A: f(p)=0\}$ for some $p \in S$. Is $A$ an algebra of measurable functions on $S$ ?

The answer is "no." The example which follows is briefly studied in $[3, \S 9]$ from a different point of view.

Example. Let $S=[0,1] \times[0,1]$, and let $A$ be those $f \in R^{S}$ for which: given $\epsilon>0$ there is a countable collection $\left\{R_{n}\right\}$ of rectangles in $S$

\footnotetext{
${ }^{2}$ This question arose during an interesting conversation with D. G. Johnson, J. Kist and S. Willard.
} 
(i.e., sets of the form $E \times F$, where $E$ and $F$ are (any) subsets of $[0,1]$ ) with $\bigcup R_{n}=S$ and $\operatorname{osc}_{R_{n}} f<\epsilon$ for each $n$.

Without difficulty one verifies that $A$ is a point-separating subalgebra of $R^{s}$, that $1 \in A$, and that $A$ is closed under inversion and uniform convergence. For $p \in S$, the characteristic function of $\{p\}$ is in $A$, so $A$ induces the discrete topology on $S$.

Now let $D$ stand for $[0,1]$ with the discrete topology, and let $\beta D$ be the Stone-Cech compactification. Because $D$ is realcompact, $D$ is " $Q$-closed" in $\beta D$, i.e., if $p \in \beta D-D$ there is a $G_{\delta}$-set $G$ of $\beta D$ with $p \in G$ and $G \cap D=\varnothing[2,8 \mathrm{~B}]$. It follows easily that $D \times D$ is $Q$-closed in $\beta D \times \beta D$ [3, Lemma 9.5]. By [3, 9.3 and 4.2], $\mathcal{R}(A)=D \times D$. Hence, if $M \in \mathcal{R}(A)$, then $M=M_{p}$ for some $p \in S$. (A direct proof of this is too lengthy to include here.)

We now show that $A$ is not an algebra of measurable functions. Let $\Delta=\{(x, x): x \in[0,1]\}$. For $f \in R^{S}$, let $Z(f)=\{s \in S: f(s)=0\}$. We claim that there is $f \in A$ with $Z(f)=\Delta$. First, there are $f_{1}, f_{2}, \cdots$ $\in A$ with $\Delta=\bigcap Z\left(f_{n}\right)$ : define $f_{n}$ by covering $\Delta$ by finitely many squares in $S$ each of side $1 / n$, and each meeting $\Delta$, let $E$ be the union of these squares, and let $f_{n}$ be the characteristic function of $S-E$. But $\bigcap Z\left(f_{n}\right)=Z\left(\sum 2^{-n} f_{n}\right)$, and $\sum 2^{-n} f_{n} \in A$. Now, it is easily checked that if $A$ were the algebra of $A$-measurable functions, then $A$ and the family $\{Z(f): f \in A\}$ would coincide. Hence, if $f \in A$, then $Z(f)$ $=S-Z(g)$ for some $g \in A$. From the definition of $A$, it follows that each $S-Z(g)$ is the union of a sequence of rectangles. Thus $\Delta$ would be the union of a sequence of rectangles, which is not possible.

Remarks. (1) The algebra in the example lives on the set $[0,1] \times[0,1]$ only for convenience. Any uncountable set would do. To obtain an algebra with the desired properties on a set of cardinal $\boldsymbol{\aleph}_{1}$, let $S_{1}=T \times T$ where $T$ has cardinal $\boldsymbol{\aleph}_{1}$, let $A_{1}$ be defined as above, and view $T$ as a subset of $[0,1]$ to achieve a contradiction in the above manner. To obtain an algebra with the desired properties on the arbitrary uncountable set $D$, let $\alpha: S_{1} \rightarrow D$ be one-to-one, and let

$$
A \equiv\left\{f \in R^{D}: f \circ \alpha \in A_{1}\right\} .
$$

On the other hand, the construction in the example would not achieve the desired contradiction were $[0,1]$ replaced by a countable set: for then the diagonal would be the union of a sequence of rectangles. Indeed, on a countable set, there is no algebra with the desired properties: if $S$ is a countable set, and $A$ is a subalgebra of $R^{S}$ as in the Question, then $A$ is all functions on $S$ which are continuous relative to the topology on $S$ which $A$ induces - this follows immediately from $[5,5.4]$; see also [3] — and this is the algebra of $A$-measurable functions, with $\mathcal{A}=\{\operatorname{coz}(f): f \in A\}$. 
(2) The algebra of the example is the algebra of all functions in $R^{S}$ which are uniformly continuous relative to the uniformity on $S$ generated by the countable covers whose members are rectangles (and the usual uniformity on $\boldsymbol{R}$ ). It is not at all clear what properties of this uniformity are "responsible for" the presently relevant properties of the algebra. More generally, what properties should a uniformity possess in order that it be associated in a natural way with an algebra of measurable functions? A study of this would be interesting. Compare [6].

(3) If $A$ is an algebra which provides a negative answer to the Question, then $\mathcal{M}(A)$, its space of maximal ideals (see [5]) is a compact space which is not basically disconnected (see $[3,2.3])$ in which each dense cozero-set is $C^{*}$-embedded (see $[4,2.3]$ or [6]) and which contains a dense $P$-subspace $(\mathcal{R}(A))$ which is $Q$-closed. Conversely, if $X$ is such a compact space, with $P$-subspace $S$, then

$$
\{f \mid S: f: X \rightarrow R \cup\{ \pm \infty\}, f \text { is continuous, } f \leftarrow(R) \supset S\}
$$

is an algebra providing a negative answer to the Question. (The desired properties follow from [3, 2.5 and 4.2] and [4, 2.3].)

Conceivably, each compact space with the above properties must be an $F$-space [2], but, in fact, I do not know if $\mathcal{M}(A)$, where $A$ is as in the example, is an $F$-space, and I know of no compact $F$-space which is not basically disconnected with a dense $Q$-closed discrete set (or $P$-subspace). And I know of no compact space with the properties of the preceding paragraph in the literature.

\section{REFERENCES}

1. F. W. Anderson, Approximation in systems of real-valued continuous functions, Trans. Amer. Math. Soc. 103 (1962), 249-271. MR 25 \#436.

2. L. Gillman and M. Jerison, Rings of continuous functions, Van Nostrand, Princeton, N.J., 1960. MR 22 \#6994.

3. A. W. Hager, On inverse-closed subalgebras of $C(X)$, Proc. London Math. Soc. (3) 19 (1969); 233-257. MR 39 \#6261.

4. —, Algebras of measurable functions, Duke Math. J. 38 (1971), 21-27.

5. M. Henriksen and D. G. Johnson, On the structure of a class of archimedean lattice-ordered algebras, Fund. Math. 50 (1961/62), 73-94. MR 24 \#A3524.

6. J. R. Isbell, Algebras of uniformly continuous functions, Ann. of Math. (2) 68 (1958), 96-125. MR 21 \#2177.

Wesleyan University, Middletown, Connecticut 06457 